\title{
Investigation of thermal processes in abrasive pipe sampling
}

\author{
Elena Levchenko ${ }^{1, *}$, and Nikolay Pokintelitsa ${ }^{1}$ \\ ${ }^{1}$ Sevastopol State University, Department of Mechanical Engineering, 299053 Sevastopol, Russia
}

\begin{abstract}
The paper presents the results of research on the process of interaction between the cutting circle and the cut pipe billet on the basis of heat-deforming analysis with the establishment of a relationship between processing conditions and cutting conditions with the design parameters of the abrasive tool.
\end{abstract}

\section{Introduction}

Abrasive cutting of materials, from the point of view of the physical processes occurring in the surface layers of the cut billets, is one of the highest-temperature and high-speed machining processes. The thermal process arising during the cut is characterized by temperatures between $200-800{ }^{\circ} \mathrm{C}$, the instantaneous heating with velocities of hundreds of thousands of degrees per second. Following rapid heating, heat is removed from the surface layers deep into the pipe material at a cooling rate of approximately the same order as in heating.

The presence of heat exchange with the environment causes the redistribution of heat fluxes in the system "cutting circle - cut pipe-chip", and as a consequence, leads to a change in the basic kinetic parameters of the thermal process. The temperature deformations of parts along the length and diameter depend on the coefficient of thermal conductivity of their material [1, 3]. It is known that heat-resistant steel $12 \mathrm{H} 18 \mathrm{~N} 9 \mathrm{~T}$ is less heatconducting than stainless steels $4 \mathrm{H} 13,3 \mathrm{H} 13$ and steel grades $08 \mathrm{kp}$ and $1 \mathrm{ps}$. However, they have about 1,5 times the large coefficient of linear expansion and, as a consequence, higher temperatures appear in the cutting zone. Such circumstances determine the propensity of heat-resistant steels to high temperature deformations exceeding the deformation of stainless steels and structural steels of grades $08 \mathrm{kp}$ and $1 \mathrm{ps}$. As is known, the quality parameters of the surface layer of the billet largely depend on the thermal factor. The physicomechanical state of the surface layer of the cut billets is determined not only by the contact temperature, which can be measured experimentally, but by the entire spacetime temperature field [1]. In particular, the gradients of temperatures and the rate of change of temperature are of paramount importance in the formation of the surface layer. All these factors have unique functional relations with the kinematic and dynamic parameters of the processing regime, the properties of the material, the characteristics of the circle, and so on. The quality control of the cut surface of the billet largely reduces to establishing these bonds and acting through them on the thermal regime of the cutting and the physical and mechanical properties of the surface layer. Therefore, the aim of this paper is to select and justify the method for determining the temperature in the treatment zone, taking into account the influence of the temperature factors accompanying the cutting process on the efficiency of the technological operation.

\section{Main text}

To determine the causes of the errors, a heat-deforming analysis of the process was carried out. For this purpose, the regularity of the temperature distribution in the billet has been established. It was assumed that the width of the groove of the workpiece increases only as a result of thermal expansion (Fig. 1).

In the process of work, the circle as a whole and its abrasive grains enter the metal of the workpiece. The cutting wheel is unevenly heated along the length of the working part.

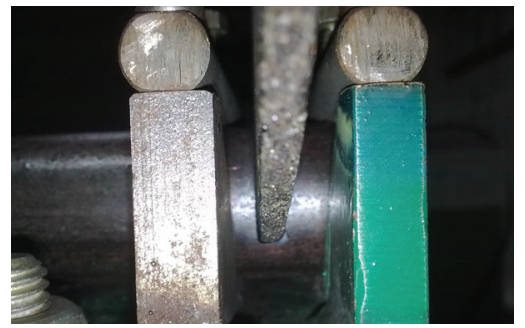

Fig. 1. Scheme of cutting the workpiece.

Since the normal components of the cutting force in abrasive cutting reach large values, the most protruding grains and the binder holding them undergo an elastic deformation and the number of grains in contact with the metal surface increases substantially. Not all contacting grains will participate in the removal of metal. But if we evaluate the grains as heat sources that heat the metal, then the grains sliding along the surface will contribute no less than those that remove the shavings. Cutting grains located on the side surfaces of the circle absorb a generally smaller part of the energy, since it is also expended on the work of deformation and heating of the formed chips.

* Corresponding author: ealev1978@mail.ru 
At the initial moment of cutting due to intense heating, the material of the workpiece substantially extends toward the resulting groove. Then, as the tool moves in the feeding direction, the linear expansion proceeds more slowly. As a result, a section of the groove is formed, the profile of which differs significantly from the nominal.

The temperature deformations of the workpiece cause the shape of the groove to distort, which it would absorb after cooling (Fig. 2). The actual shape of the groove section is obtained taking into account the thermal deformations of the workpiece.

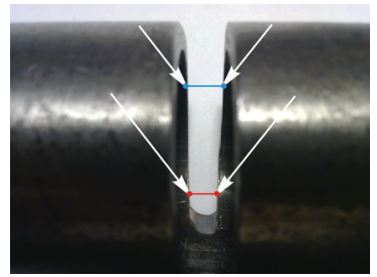

Fig. 2. Schematic of the change of the groove under Exposure to temperature deformations.

As shown by experimental studies [4], the process of mass cutting of abrasive grains during cutting, proceeding at very high speeds, creates a large number of high-temperature centers in the surface layer. The sources of heat arising during the cutting are the work of metal deformation and the work of external friction of abrasive grains on the metal surface. In contrast to cutting with a metal tool in abrasive cutting, the heat of cutting is mainly transferred to the mass of the metal of the part, the cutting wheel is practically not heatconductive. Knowing the cutting temperatures and the thermal phenomena accompanying this process is necessary not only to obtain a qualitative surface layer, but also to establish the true picture of the process of removing the thinnest chips at high speeds.

It is known that the process of heat transfer in a stationary solid medium is carried out due to heat conductivity and is described by the equation [2]

$$
\frac{d \Theta}{d t}=\frac{\lambda}{C \gamma}\left(\frac{\partial^{2} \Theta}{\partial x^{2}}+\frac{\partial^{2} \Theta}{\partial y^{2}}+\frac{\partial^{2} \Theta}{\partial z^{2}}\right) .
$$

In the presence of motion of a heat source, it is necessary to associate a coordinate system with a moving source. When the $\mathrm{x}$-axis is aligned with the direction of motion of the heat source, the differential heat equation (1) is written in the form

$$
\frac{d \Theta}{d t}=\frac{\lambda}{C \gamma}\left(\frac{\partial^{2} \Theta}{\partial x^{2}}+\frac{\partial^{2} \Theta}{\partial y^{2}}+\frac{\partial^{2} \Theta}{\partial z^{2}}\right)+V \frac{d \Theta}{d x} .
$$

where $V-$ is the velocity of the heat source, $\mathrm{m} / \mathrm{s} ; \mathrm{C}-$ is the specific heat, $\mathrm{J} /(\mathrm{kg} \cdot \mathrm{K}) ; \gamma$ - density, $\mathrm{kg} / \mathrm{m}^{3} ; \lambda$ - is the coefficient of thermal conductivity, $\mathrm{W} /(\mathrm{m} \cdot \mathrm{K})$.

To solve thermophysical problems in abrasive metal cutting processes, the source method (Green's method) is used, the essence of which is that if the law of temperature variation $\Theta(x, y, z, t)$ at point $\mathrm{B}(x, y, z)$ is known ) Of the heat-conducting space from the heat source placed in the point $A\left(x^{\prime}, y^{\prime}, z^{\prime}\right)$, which allocated heat quantity $\mathrm{Q}$ at time $t^{\prime}$, then the temperature at point $\mathrm{B}$ $(x, y, z)$ from the system of spatially distributed sources Is determined by superposition of temperature fields. The solution of the problem reduces to determining the function $\Theta(x, y, z, t)$ from the heat source $\mathrm{Q}$ (Green's function). In order for the solution $\Theta(x, y, z, t)$ to be the unique solution of the problem, it must simultaneously satisfy certain initial and boundary conditions.

The initial condition $\Theta=\Theta\left(x, y, z, t_{0}\right)$ determines the temperature distribution inside the investigated region at the initial instant of time.

The boundary conditions in the theory of thermal conductivity are divided into four types.

1. The boundary conditions of the first kind determine the temperature distribution at the boundary of the region at any instant of time, i.e. $\Theta\left(x_{S}, y_{S}, z_{S}, t\right)=\varphi$ $(t, S)$.

2. A boundary condition of the second kind determines the density of the heat flux for each point of thermal space at any instant of time, i.e. $q\left(x_{S}, y_{S}, z_{S}, t\right)$.

3. A boundary condition of the third kind determines the heat exchange at the boundary of the region with the surrounding medium, which can be specified in the form of the Newton-Richman law:

$$
q=\alpha\left(\Theta_{O}-\Theta_{C}\right) ; \alpha\left(\Theta_{O}-\Theta_{C}\right)+\left.\lambda \frac{\partial \Theta}{\partial n}\right|_{S}=0,
$$

where $\Theta_{O}$ - is the surface temperature of the workpiece; $\Theta_{C}$ - ambient temperature; $\alpha$-is the coefficient of heat exchange, depending on the properties of the material of the workpiece, medium and temperature.

4. The boundary conditions of the fourth kind express the equality of the heat fluxes at the interface between two heat-conducting media and are written in the form of the equation:

$$
-\left.\lambda_{1} \frac{\partial \Theta_{1}}{\partial x}\right|_{S}=\left.\lambda_{2} \frac{\partial \Theta_{2}}{\partial x}\right|_{S} .
$$

The distribution of thermal sources in space, the time of their action and motion are taken into account by integration.

The fundamental solution of the heat equation (2) is the source function with a continuously varying coordinate $x^{\prime}$ according to the $\operatorname{law}\left(x^{\prime}-V t\right)$. Fig. 3 shows a thermal source moving in the XOY plane together with the moving coordinate axes [2].

At the initial instant of time, the source $\tau=t^{\prime}$ occupies a position at a point $A\left(x^{\prime}-V\left(t-t^{\prime}\right), y^{\prime}\right)$, and at the final instant of time - at a point $A^{\prime}\left(x^{\prime}, y^{\prime}\right)$. When moving the coordinate axes by a distance $V\left(t-t^{\prime}\right)$, the temperature at the point $B(x, y)$ is equal to the superposition from all positions of the moving heat source. When you move the coordinate axes, the square 
of the distance $R^{2}$ changes also in the final position of the coordinates axes (for $\tau=t$ ) at the point $A^{\prime}\left(x^{\prime}, y^{\prime}\right)$ :

$$
R_{\kappa}^{2}=\left(x-x^{\prime}\right)^{2}+\left(y-y^{\prime}\right)^{2},
$$

And in the initial position of the coordinate axes at the point $A\left(x^{\prime}-V\left(t-t^{\prime}\right), y^{\prime}\right)$ :

$$
R_{H}^{2}=\left[x-x^{\prime}+V\left(t-t^{\prime}\right)\right]^{2}+\left(y-y^{\prime}\right)^{2} .
$$
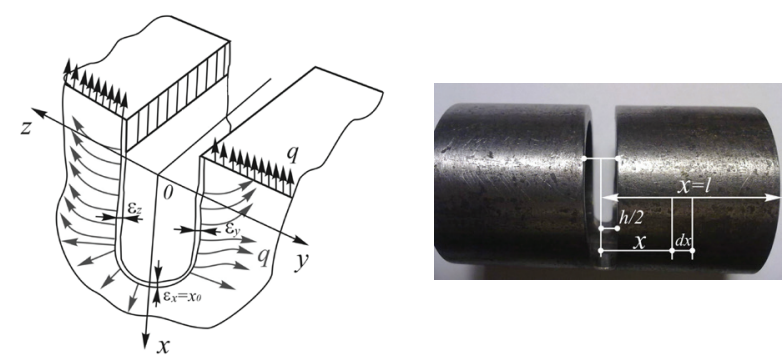

Fig. 3. Diagram of the movement of the heat source.

It is known that if in the n-dimensional space at the point A with the coordinates $x_{1}^{\prime}, x_{2}^{\prime}, x_{3}^{\prime}, \ldots, x_{n}^{\prime}$ at the time $t^{\prime}$, the amount of heat $Q$ is released, then the body temperature at point $\mathrm{B}$ with the coordinates $x_{1}, x_{2}, \ldots, x_{\mathrm{n}}$ at time $\left.\left(t>t^{\prime}{ }_{n}\right)\right)$ is described by the function [1]

$$
\begin{aligned}
& \Theta\left(x_{1}, x_{2}, \ldots, x_{n}, x_{1}^{\prime}, x_{2}^{\prime} \ldots x_{n}^{\prime}, t, t^{\prime}\right)= \\
& =\frac{\mathrm{Q}}{C \gamma\left[\sqrt{4 \pi a\left(t-t^{\prime}\right)}\right]} \cdot \exp \left\{-\frac{R^{2}}{4 a\left(t-t^{\prime}\right)}\right\},
\end{aligned}
$$

where $R^{2}$ - is the square of the distance between points $\mathrm{A}$ and $\mathrm{B}$,

where $R^{2}=\left(x_{1}-x_{1}^{\prime}\right)^{2}+\left(x_{2}-x_{2}^{\prime}\right)^{2}+\ldots+\left(x_{n}-x_{n}^{\prime}\right)^{2}$; $a-$ is the coefficient of thermal diffusivity, $\mathrm{m}^{2} / \mathrm{s}$.

The function (3) is the solution of the fundamental heat equation (2). On the basis of this solution, we can write down the Green's function for various conditions of the problem.

As is known, the quality parameters of the surface layer of the billet largely depend on the thermal factor. If the cutting conditions and other processing conditions change in such a way that the amount of heat generated in the cutting zone increases, then the shape and size of the cutting groove should be expected to change.

On the basis of the foregoing, the issue of heat distribution between contacting bodies should be decided on the basis of the thermal contact between the two bodies: the cut billet and the abrasive wheel.

Consider the question of the proportion of heat going into the cutting circle. This task must be solved from the prediction of the discreteness of contact between the circle and the workpiece. If for a cut surface the rapid change of thermal impulses on the most active grains merges into a continuous background, then for the cutting circle the contact is always discrete, acting for a short time, depending on the speed of the circle. Advantageous heat dissipation on grains in comparison with a binder makes it possible to model each grain in the form of a half-space having a contact in a small area with another half-space moving relative to the first with the speed of supply of the lateral surfaces of the circle. The question of the distribution of heat between these half-spaces is solved on the basis of the equality of average contact temperatures. This condition [5 - 11] will lead to a formula for determining the input coefficient $\alpha_{b}$, which shows how much heat goes into the half-space (with respect to abrasive cutting is the coefficient $\alpha_{b}$ that shows how much heat goes into the workpiece):

$$
\alpha_{b}=\frac{1}{1,25 \frac{\lambda_{\kappa}}{\lambda} \sqrt{\frac{a}{d_{u} \cdot V_{\kappa}}}+1},
$$

where $\lambda_{\kappa}-$ coefficient of thermal conductivity of the cutting circle, $\mathrm{W} /(\mathrm{m} \cdot \mathrm{K}) ; \lambda-$ coefficient of heat conductivity of the workpiece material, $\mathrm{W} /(\mathrm{m} \cdot \mathrm{K}) ; a-$ coefficient of thermal diffusivity of the billet material, $\mathrm{m}^{2} / \mathrm{s} ; V_{\kappa}$ - speed of the cutting wheel, $\mathrm{m} / \mathrm{s} ; d_{3}$ - average diameter of the contact area of the cutting grain with the workpiece (wear surface on the grain), $\mathrm{m}$.

This form of writing for the input coefficient is convenient for analysis, since the numerical value $\alpha_{b}$ can be judged from the size of the dimensionless complex in the denominator as compared to unity. With abrasive cutting at all practical regimes, the complex is considerably less than unity.

$$
1,25 \frac{\lambda_{\kappa}}{\lambda} \sqrt{\frac{a}{d_{u} \cdot V_{\kappa}}} .
$$

If we take into account that for most abrasive materials the heat conductivity is ten times less than the thermal conductivity of metals, the most approximate estimates show that the fraction of heat going into the circle can be neglected. In fact, if we set the values most typical for the cut for all the values entering into expression (5), for example, for steel 08kp:

$$
\begin{gathered}
\frac{\lambda_{\kappa}}{\lambda}=0,1 ; a=8 \cdot 10^{-6} \mathrm{~m}^{2} / \mathrm{s} ; d_{u} \approx 0,1 \mathrm{~mm} ; \\
V_{\kappa}=80 \mathrm{~m} / \mathrm{s},
\end{gathered}
$$

We get

$$
1,25 \cdot 0,1 \sqrt{\frac{8 \cdot 10^{-6}}{0,1 \cdot 10^{-3} \cdot 80}}=0,4 \cdot 10^{-2} .
$$

Substituting this value into expression (4), we obtain that $\alpha_{B} \approx 0,99$, i.e., the circle receives no more than $1 \%$ of the total heat. Therefore, when calculating the contact temperature in the cut-off zone, the fraction of heat going into the cutting circle can be neglected. 
The total density of the heat flux formed in the cutting zone

$$
q=\frac{P_{z} \cdot V_{\kappa}}{F_{\kappa}},
$$

where $P_{z}$ - is the tangential component of the total cutting force, $\mathrm{N} ; V_{\kappa}-$ speed of the cutting wheel, $\mathrm{m} / \mathrm{s} ; F_{\kappa}-$ contact area of the circle and the cut workpiece, $\mathrm{m}^{2}$.

Using the principle of superimposing temperature fields from elementary sources of heat with allowance for integration in space and time, it is possible to determine the temperature field of a source arbitrarily distributed in space and acting for any time interval according to the formula [3]:

$$
\Theta(x)=\frac{q}{2 C \gamma \sqrt{\pi a \tau}} \exp \left[-\frac{(x-h / 2)^{2}}{4 a \tau}\right],
$$

where $C-$ is the specific heat of the metal being cut, $\mathrm{J} /(\mathrm{Kg} \cdot \mathrm{K}) ; h / 2-$ the initial size of the groove half of the cut, $\mathrm{mm} ; x$ - distance from the center line of the groove to the section in question, $\mathrm{mm} ; \tau$ - cutting time, $\mathrm{s} ; \gamma-$ density of the material of the cut billet, $\mathrm{kg} / \mathrm{mm}^{3}$.

Fig. 4 shows a graph of temperature variation along the length of the workpiece when cutting a pipe billet from steel $08 \mathrm{kp}$.

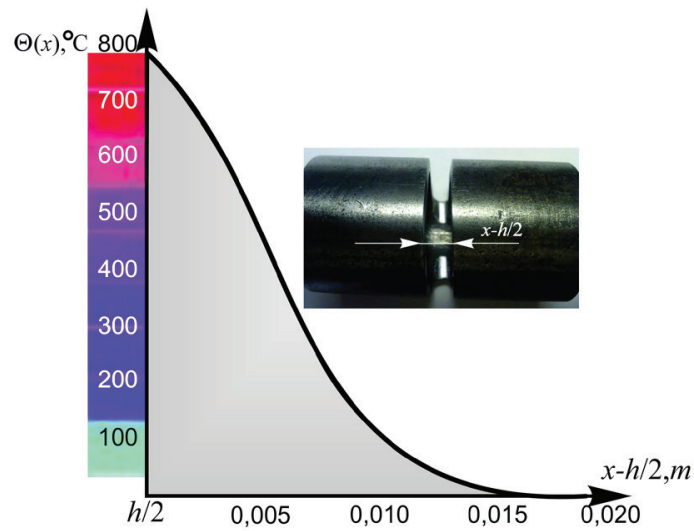

Fig. 4. Graph of temperature change along the length of the workpiece.

$d_{3}=42,4 \mathrm{~mm}$, workpiece material - steel $08 \mathrm{kp}$, circle $400 \times 4 \times 32,14 \mathrm{AF} 30$

It can be seen from Fig. 4 that the temperature varies along an extremal curve with a maximum of its values at a certain distance from the contact zone of the tool and the workpiece. This location of the maximum on the curve $\Theta=f(x)$ corresponds to the thermophysical nature of the processes occurring in the contact zone. As the source of heat generation moves, localization of the process of contact plastic deformation and, consequently, an increase in the intensity of heat release per unit volume of metal with the approach of the source of heat release and the contact surface. At a distance $x=C_{1}$, there is a transition from the plastic flow to the boundary friction and the change of the heat source from the volumetric to the source, in which heat release occurs in a thin boundary layer. Further, as the displacement along the contact increases, the intensity of heat release decreases. As a result, the following pattern is formed. Within the limits of plastic contact, the intensity of heat release in the zone of contact plastic deformations is higher than the intensity of heat sink into the tool. Therefore, there is an accumulation of heat and the temperature $\Theta(x)$ rises. At the contact site, the picture changes: the intensity of heat flow from the boundary friction zone to the tool is higher than the heat dissipation rate, so the temperature $\Theta(x)$ in this section decreases.

\section{Conclusion}

Analyzing the above mentioned, we can conclude that the process of abrasive cutting is accompanied by the release of a large amount of heat, which leads to the formation of a defect layer on the surface of the cut. It is established that the contact of the sides of the circle with the metal is due to the expansion of the material of the workpiece under the influence of heat generated by the cutting, the oscillation of the circle height and the nonuniformity of wear of its lateral surfaces.

The prospect of further research is to determine the kinematic parameters of the abrasive cutting process and their relationship to the geometry of the working surface of the cutting wheel.

\section{References}

1. V.A. Sipailov, Thermal processes during grinding and surface quality control (Mechanical Engineering, Moscow, 1978)

2. A.V. Yakimov, Fundamentals of thermal phenomena in the grinding of machine parts (OGPU, Odessa, 1997)

3. A.V. Yakimov, P.T. Slobodyanik, A.V. Usov, Thermophysics of machining (Lybid, Odessa, 2010)

4. Yu.K. Novoselov, Dynamics of surface shaping in abrasive processing (SevNTU, Sevastopol, 2012)

5. A.M. Tikhontsov, S.I. Chukhno, A.N. Korobochka, Thermal processes in the machining of materials by cutting (UMK VO, Kiev, 2012)

6. S.A. Vasin, Cut materials: Thermomechanical approach to the system of interrelations in cutting (MGTU, Moscow, 2001)

7. A.N. Reznikov, Thermal processes in technological systems (Mechanical Engineering, Moscow, 1990)

8. N.S. Penkin, A.N. Penkin, V.M. Serbin, Fundamentals of tribology and tribotechnology (Mashinostroenie, Moscow, 2008)

9. V.B. Strutinskiy, V.M, Drozdenko, Dynamic processes in machine tools (Osnova-Print, Kiev, 2010) 
10. V.F. Terentyev, Cyclic strength metallic materials (Mashinostroenie, Moscow, 2001)

11. C. Duan, M. Wang, Some metallurgical aspect of chips formed in high speed machining of high strength low alloy steel, J. Sci. Scripta Materialia. 52, 1001-1004 (2005) 